\title{
Frictional convergence at coastlines
}

\author{
By J. C. ROELOFFZEN, W. D. VAN DEN BERG and J. OERLEMANS, Institute of Meteorology \\ and Oceanography, University of Utrecht, Princetonplein 5, Utrecht 2506, The Netherlands
}

(Manuscript received July 29; in final form December 30, 1985)

\begin{abstract}
The coastline generally represents a marked discontinuity in surface roughness. The resulting mechanical forcing leads to a secondary circulation in the boundary layer, and consequently to a vertical motion field that may have a strong influence on the weather in the coastal zone. In potentially unstable air masses, frictional convergence may cause a more-or-less stationary zone of heavy shower activity, for example.

In this paper, we present a calculation of secondary flow patterns forced at a roughness discontinuity, as a function of the geostrophic wind. A neutral boundary layer is studied, with homogeneous conditions along the coastline (i.e., we study the circulation in a plane perpendicular to the coastline). The closure scheme for the turbulent mixing of momentum is based on a calculation of the eddy kinetic energy density (the $l, E$ closure). The equations are solved on a grid stretched in both the vertical and horizontal directions, with largest resolution at the roughness discontinuity. Various relaxation techniques are combined to give an efficient calculation of the stationary flow fields.

Upward motion turns out to be most pronounced when the geostrophic wind makes a small $\left(\sim 20^{\circ}\right)$ angle with the coastline (in a clockwise direction), and not when the geostrophic wind is perpendicular to the coastline, as is sometimes mentioned. The asymmetry relative to the normal to the coastline is due to the Coriolis acceleration, and not a nonlinear effect. Nevertheless, nonlinear effects are important, because they create a tendency to frontogenesis in the case mentioned above. This is shown by a comparison of the linear and nonlinear solution.

We finally present an example of heavy shower activity in the coastal zone of Belgium and The Netherlands, which is apparently due to frictional uplift and frontogenesis in a maritime polar air mass hitting the coastline at the critical angle referred to above.
\end{abstract}

\section{Introduction}

The meteorology of the coastal zone deserves special attention for two reasons. In the first place, coastal zones are heavily populated with many offshore activities going on. Most of these activities depend in some way on weather conditions, and thus on the quality of local weather forecasts. Secondly, the coastline is of large interest from a scientific point of view, because it represents a sudden change in boundary conditions to atmospheric flow. By studying the resulting effects, one may learn about some aspects of atmospheric boundary layers.

Of all meteorological phenomena typical for coastal regions, the fair weather sea-breeze circu- lation has probably been studied most extensively, e.g., Estoque (1962), Walsh (1974), Pielke (1974), Pearson et al. (1983). In contrast to this thermally-driven circulation, the mechanical forcing due to the discontinuity in surface roughness may create circulation patterns of similar amplitude and scale. Frictional convergence is mentioned in some studies as the cause of increased precipitation in coastal zones under specific conditions, e.g., Bergeron (1949), Timmerman (1963), Oerlemans (1980), but we have the impression that its effect is generally underestimated.

In boundary-layer meteorology, the growth of an internal boundary layer from a discontinuity in surface roughness has of course been studied, 
e.g., Echols and Wagner (1972), Rao et al. (1974), Schwiesow and Lawrence (1982). Such studies are concerned with the small-scale adjustment of the wind field, and in most cases, Coriolis effects are not considered. However, for problems like the influence of coastal frictional convergence on the occurrence of showers, it is important to consider the adjustment of wind speed as well as wind direction. Taylor (1969) considered both effects, for the case of a geostrophic wind perpendicular to the discontinuity in surface roughness. His results showed that adjustment of wind speed is a much faster process than adjustment of wind direction (typical length scales for a $10 \mathrm{~m} / \mathrm{s}$ geostrophic wind: $20 \mathrm{~km}$ and $500 \mathrm{~km}$, respectively). Another result of his study was the occurrence of overshoot in both wind speed and direction. With these findings in mind, it is obvious that the dependence of the frictionallyinduced flow pattern of the geostrophic wind direction may be quite complicated, in particular when the eddy diffusivity is allowed to depend on the local wind shear.

Without doing detailed calculations, one can already anticipate the occurrence of four basic types of frictional convergence. This is illustrated in Fig. 1. The coastline (or, in general terms, the surface discontinuity) always runs from south to north to avoid misinterpretations of the results.

In the case of a southwesterly geostrophic flow, decreasing wind speed over land will cause upward motion. Backing of the wind, occurring further inland, also contributes to the rising of air parcels. This type is referred to as type-1. When the flow is southeasterly, acceleration over sea leads to downward motion, and the veering of the wind to upward motion (type-2). Type-3 refers to northeasterly flow, in which case, both acceleration and veering contribute to produce downward motion. Northwesterly flow, type-4, again has opposite effects: deceleration of the flow leads to convergence, and the backing to divergence.

It is important to note from this simple qualitative reasoning that a marked asymmetry shows up. In the case of southwesterly flow, upward motion will be stronger than in the case of northwesterly, although the onshore component is the same.

Things will become more complicated when the flow is almost parallel to the coastline. Time scales for adjustment of the flow field may be long, and closed circulations (in the vertical plane perpendicular to the coast line) may occur.
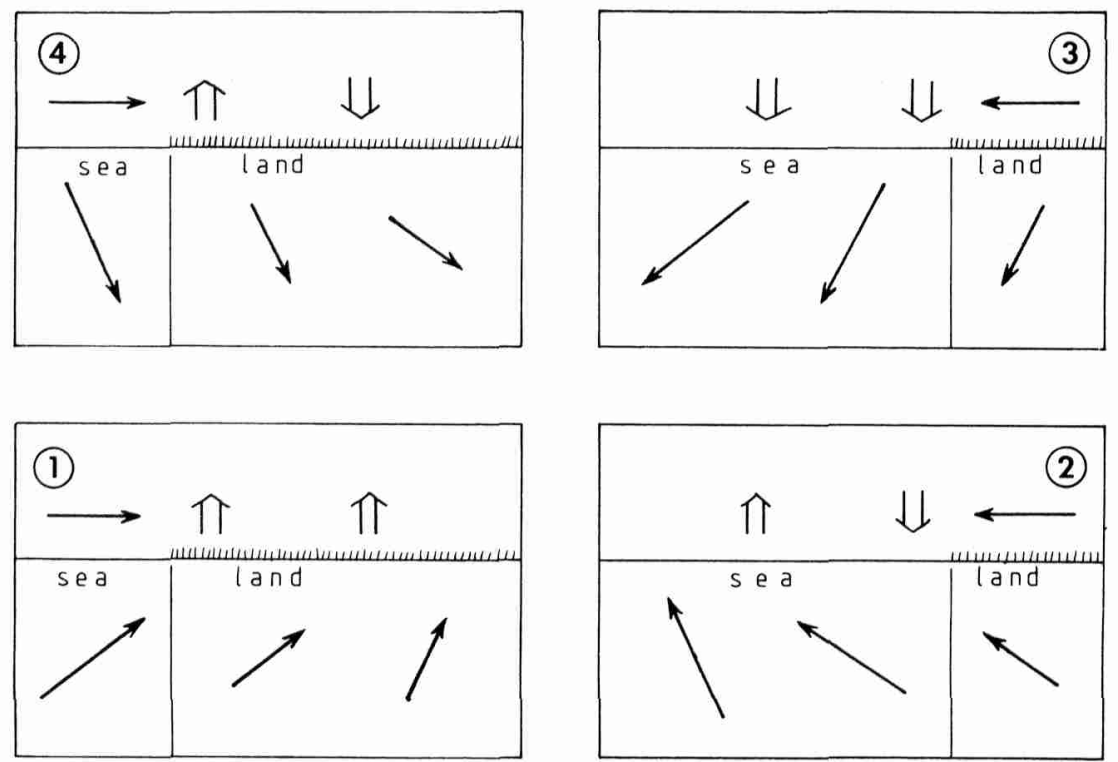
Numerical models are thus needed to see how frictional convergence is established in such situations.

At first sight, it seems that present-day mesoscale models (for a review, see Anthes (1983)) should be able to clarify this point. One could run them to equilibrium for many directions of the geostrophic flow and compare the secondary flow patterns. Such a procedure has its problems, however. To resolve the discontinuity in surface roughness, small grid distances are required (a few hundred meters, say). Since turning of the wind is slow, a large computational domain is nevertheless needed (hundreds of kilometers). The only efficient way to deal with this problem is to use a stretched coordinate system, which most mesoscale models don't have. Even if this modification to an existing model would have been made, computational times would be very large because of the large number of grid points and slow damping to a steady state (unless unrealistically large diffusion is included).

Based on these considerations, we decided to develop a model that could produce stationary flow patterns, for any geostrophic wind, in a more efficient way. To obtain solutions to the appropriate steady-state equations, a relaxation method was designed that, after solving a number of numerical problems, turned out to be far more efficient than time integration. It was therefore also possible to use the $l, E$ closure for the turbulent mixing of momentum (eddy exchange coefficient depends on mixing length $l$ and eddy kinetic energy $E$; for a review, see Detering (1985)).

Stationary flow patterns for many directions of the geostrophic wind are presented in Section 3. All calculations were carried out for a neutral stratification. This may appear restrictive, but it keeps the problem relatively simple. Nevertheless, it turns out that even for neutral conditions, the variation of the secondary flow with geostrophic wind direction is quite complicated. Results are mainly presented by streamlines in a vertical plane perpendicular to the coast, because this directly shows the vertical displacement of air parcels, which is most relevant for potentially unstable air masses.

Dramatic effects occur when the geostrophic wind is onshore and makes a small angle $\left(\simeq 20^{\circ}\right)$ with the coastline. Confluence then occurs over a substantial depth of the boundary layer with consequent frontogenesis and a small zone of large upward velocity (this result was in fact discussed by Bergeron, (1949)). There is ample evidence that the formation of showers and activity of synoptic-scale fronts is strongly influenced by frictional convergence when the geostrophic wind is "just onshore". We give some examples for The Netherlands in Section 4, which, in our view, give credit to the theory of frictional convergence developed in this paper.

\section{Description of the model}

All calculations were done for a neutrally stratified atmosphere. Geostrophic wind speed and direction, and the step in surface roughness are the only external parameters. Incorporation of thermodynamics would make the model much more complicated and goes beyond the scope of the present study.

The formulation of the basic equations essentially follows $\mathrm{Ng}$ and Spalding (1972), with empirical constants taken from Blackadar (1962), Yu (1977) and Nieuwstadt and Driedonks (1979). We adapt a coordinate system with $x$ in the easterly direction, $y$ in the northerly direction (coastline: $x=0$ ), and $z$ pointing upwards. The velocity field is assumed to be independent of $y$. For the stationary case, we then have the following equations:

$$
\begin{aligned}
& u \frac{\hat{\partial} u}{\partial x}+w \frac{\partial u}{\partial z}=f\left(v-v_{\mathrm{g}}\right)+\frac{\hat{c}}{\hat{c} z}\left(K_{m} \frac{\hat{\partial} u}{\hat{c} z}\right), \\
& u \frac{\partial v}{\partial x}+w \frac{\hat{c} v}{\hat{c} z}=-f\left(u-u_{\mathrm{g}}\right)+\frac{\hat{c}}{\partial z}\left(K_{m} \frac{\hat{\partial} v}{\partial z}\right), \\
& \frac{\hat{c} u}{\hat{c} x}+\frac{\hat{c} w}{\hat{c} z}=0, \\
& u \frac{\hat{c} e}{\partial x}+w \frac{\hat{c} e}{\hat{c} z}=K_{m}\left[\left(\frac{\partial u}{\partial z}\right)^{2}+\left(\frac{\partial v}{\partial z}\right)^{2}\right] \\
& \quad+\frac{\hat{c}}{\hat{c} z}\left(K_{r} \frac{\hat{\partial} e}{\partial z}\right)-\frac{(c e)^{3 / 2}}{l} .
\end{aligned}
$$

Eqs. (1) and (2) are the momentum equations in which $u, v$ and $w$ are the wind components in the $x, y$ and $z$ directions, respectively. The subscript $\mathrm{g}$ indicates the geostrophic wind, and $f$ is the Coriolis parameter $\left(10^{-4} \mathrm{~s}^{-1}\right.$ in this study). The Reynolds stresses are treated through the mixinglength theory; the exchange coefficient is denoted 
by $K_{m}$. Eq. (3) states that the flow is considered to be incompressible.

As has been done by many workers, the exchange coefficient is related to the eddy kinetic energy $e$, the budget of which is formulated in eq. (4). Here, the advection of $e$ balances the production from the shear (first term on righthand side), diffusion (parameterization of divergence of eddy fluxes and pressure fluctuation term) and dissipation (last term). For a general discussion on the kinetic-energy budget equation and the interpretation of the various terms, we refer to Tennekes and Lumley (1972).

In eq. (4), three new parameters have been introduced, namely, the exchange coefficient for $e\left(K_{e}\right)$, a constant $c$, and mixing length $l$ of the turbulent eddies. For the latter we use (e.g., $\mathrm{Ng}$ and Spalding, 1972):

$\frac{1}{l}=\frac{1}{R Z}+\frac{1}{\lambda}$

Here $R$ is Von Karmann's constant (0.4). For the limiting length scale $\lambda$, we follow Blackadar (1962) and $\mathrm{Yu}(1977)$ :

$\lambda=\alpha\left(u_{\mathrm{g}}^{2}+v_{\mathrm{g}}^{2}\right)^{1 / 2} / f$,

with $\alpha=0.00027$. In addition, $K_{u}$ was set to 0.8 times $K_{m}$, which is the optimal value proposed by Yu (1977).

Finally, the mixing coefficient $K_{m}$ is related to the turbulent kinetic energy through:

$K_{m}=l \sqrt{c e}, \quad c=0.2$

We now turn to the boundary conditions. The laminar sublayer and buffer layer are not treated, so the logarithmic layer is in fact the lowest layer represented in the model. Straightforward boundary conditions for momentum then are:

$u\left(x, z_{0}\right)=0, \quad v\left(x, z_{0}\right)=0$,

$\lim _{\Sigma \rightarrow,} u=u_{\gamma}, \quad \lim _{z \rightarrow,} v=v_{\gamma}$

$\lim _{x \rightarrow \pm} \frac{\partial u}{\partial x}=\lim _{x \rightarrow \pm} \frac{\partial v}{\partial x}=0$.

For the eddy-kinetic energy, we use

$\left.\frac{\hat{c} e}{\hat{c} z}\right|_{\bar{z}_{0}}=0, \quad \lim _{z \rightarrow+} e=0$,

$\lim _{x \rightarrow \pm,} \frac{\partial e}{\partial x}=0$.

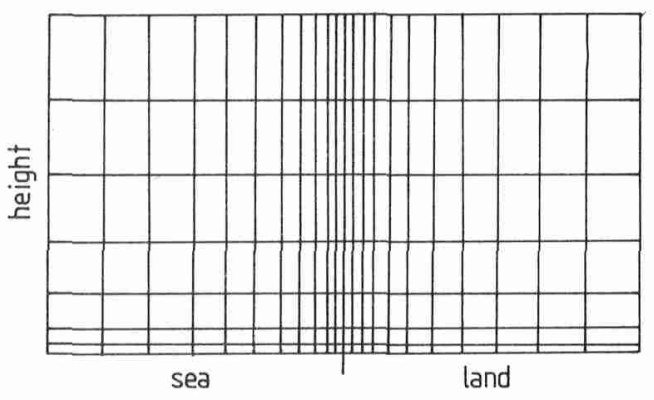

Fig. 2. Schematic representation of the grid. In the vertical and horizontal directions, the coordinate axes are stretched away from the discontinuity in surface roughness.

At this point, the basic model has been formulated. However, because it is desirable to have a better resolution in the numerical model near the surface, a new vertical coordinate $\zeta$ is introduced:

$z=\mathrm{e}^{\zeta / 5^{\circ}}$.

It can also be expected that gradients are largest near the discontinuity in surface roughness, so it makes sense to stretch the horizontal coordinate away from $x=0$. This is done by using a coordinate $\zeta$, which relates to $x$ as:

$|x|=b\left(\mathrm{e}^{\zeta}-1\right)$.

The degree of stretching is thus given by the constants $\zeta^{*}$ and $b$. A typical example of a resulting grid is shown in Fig. 2.

For all computations reported in this paper, we use a grid of 20 points in the vertical and 63 points in the horizontal direction. The size of the model domain is $3000 \mathrm{~m}$ in the vertical and runs from -1000 to $+1000 \mathrm{~km}$. For given geostrophic wind and difference in surface roughness, the solution is calculated using the Gauss-Seidel point over-relaxation.

\section{Results of the numerical model}

Stationary flow patterns were calculated for many wind directions. The roughness length over sea was set to $10^{-4} \mathrm{~m}$, over land to $0.1 \mathrm{~m}$. The basic series of calculations was done with a 20 $\mathrm{m} / \mathrm{s}$ speed of the geostrophic wind. 


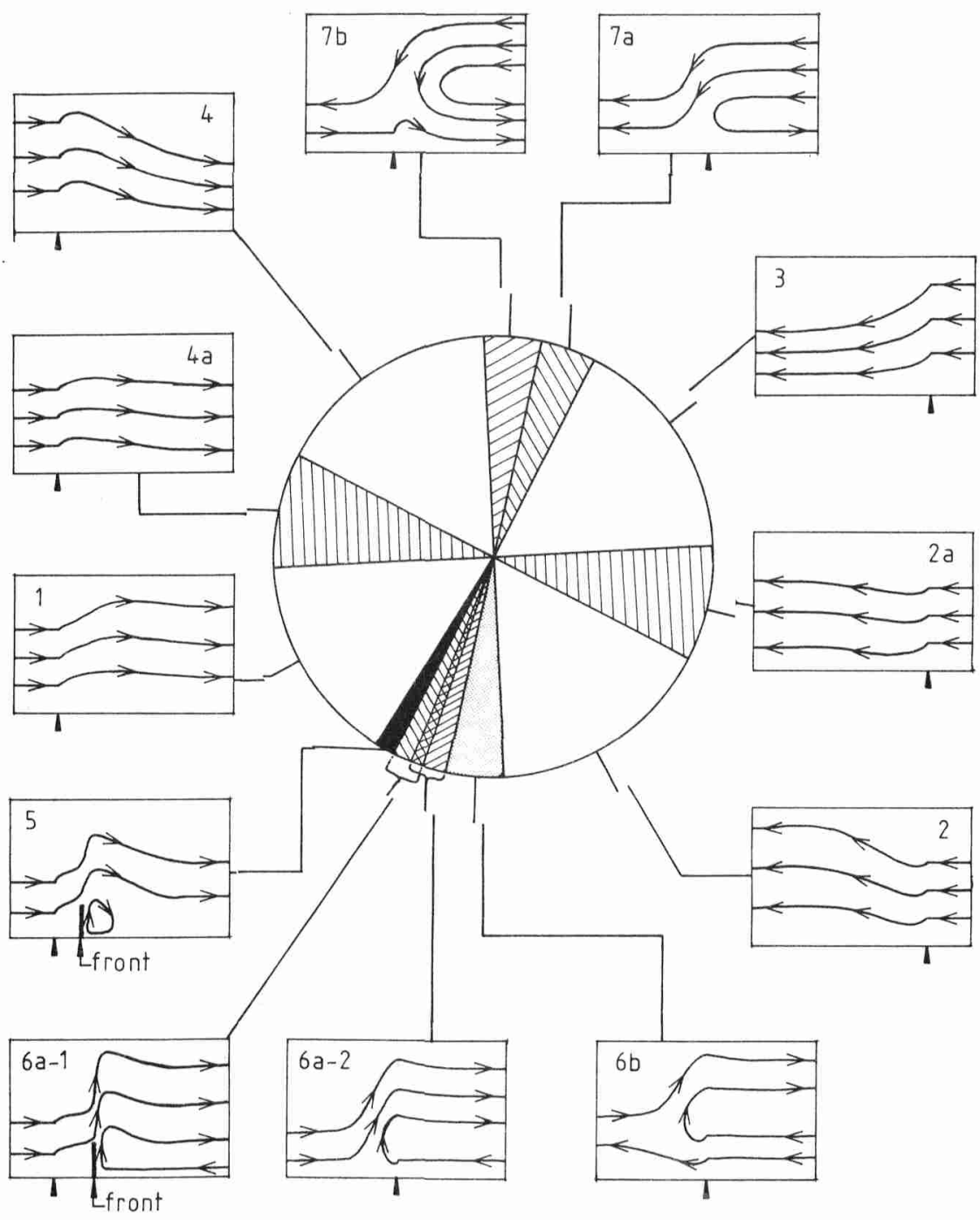

Fig. 3. Overview of the calculated flow regimes, as a function of the geostrophic wind direction. The coast line runs from the top to the bottom of the figure, while the sea is at the left-hand side. The corresponding geostrophic wind vector points from the edge to the centre of the figure. Different shadings correspond to qualitatively different flow patterns; the numbers refer to the discussion in the text. The curves shown in each panel represent trajectory projections on the plane perpendicular to the coastline.

An overview of the results is given in Fig. 3, and more detailed flow patterns are displayed in Fig. 4.

The flow patterns are shown in terms of projections of streamlines on the vertical plane perpendicular to the coastline. A division is made into patterns that have qualitative differences.
The asymmetry mentioned in Section 1 clearly shows up. Compare, for instance, northwesterly flow (deceleration $\rightarrow$ upward motion, backing $\rightarrow$ downward motion) with southwesterly flow (deceleration and backing $\rightarrow$ upward motion).

Complicated stream patterns occur when the geostrophic wind blows almost parallel to the 

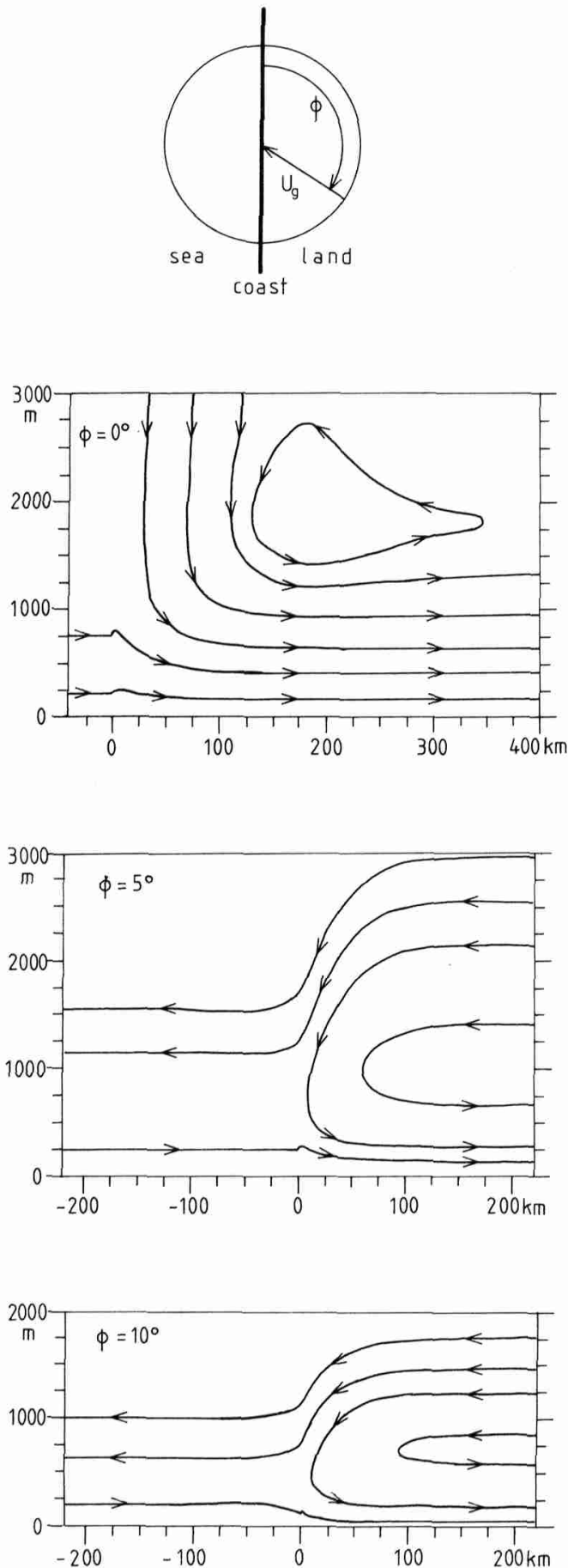
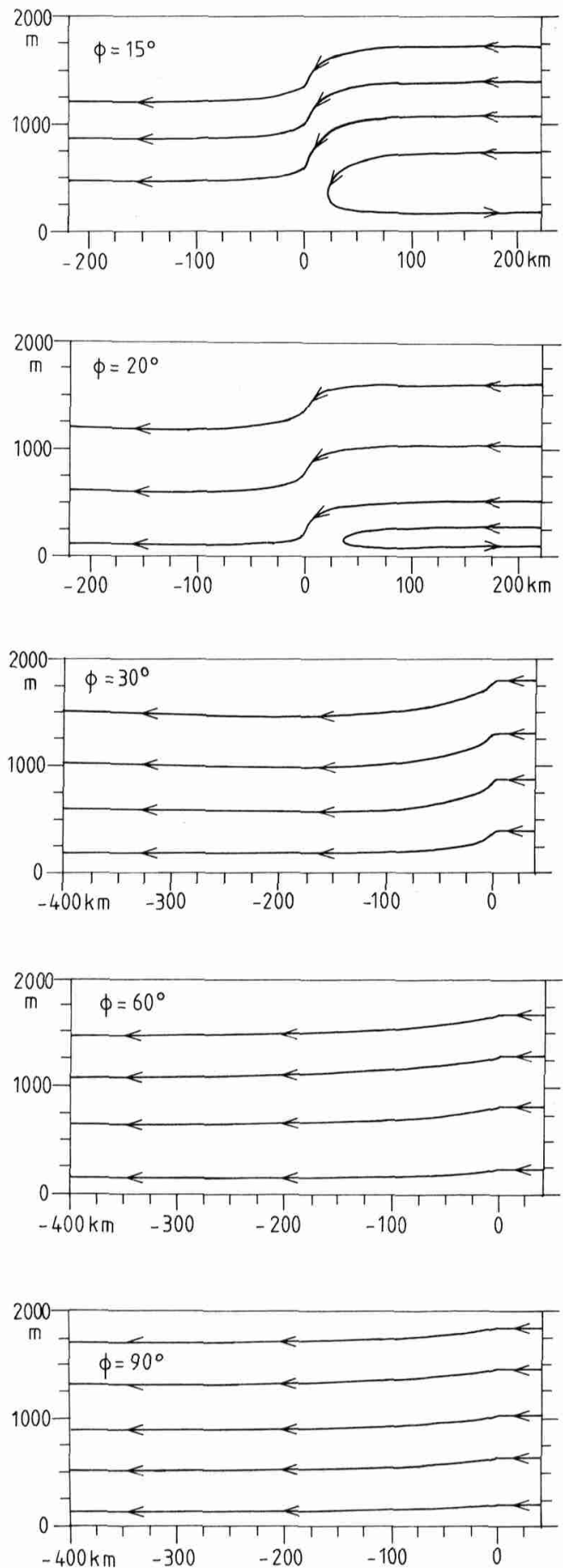

Fig. 4. Computed flow patterns at different geostrophic wind directions. The geostrophic wind speed is $20 \mathrm{~m} / \mathrm{s}$. The figures give the projections of some selected trajectories. Note that the density of the lines is not a measure of the flow intensity! The definition of the wind direction $\phi$ is shown in (a). 

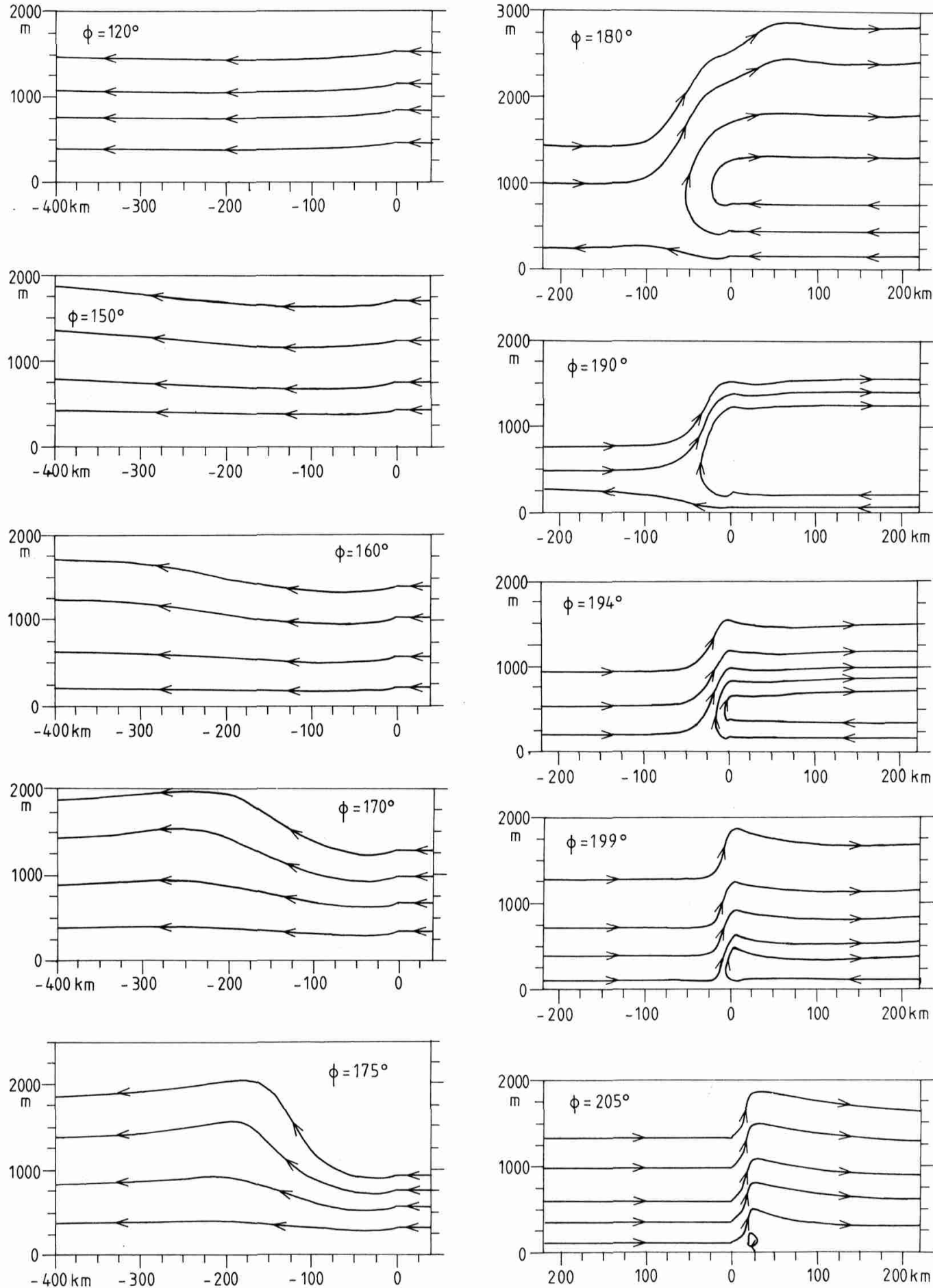

Fig. 4 (continued).

Tellus 38A (1986), 5 

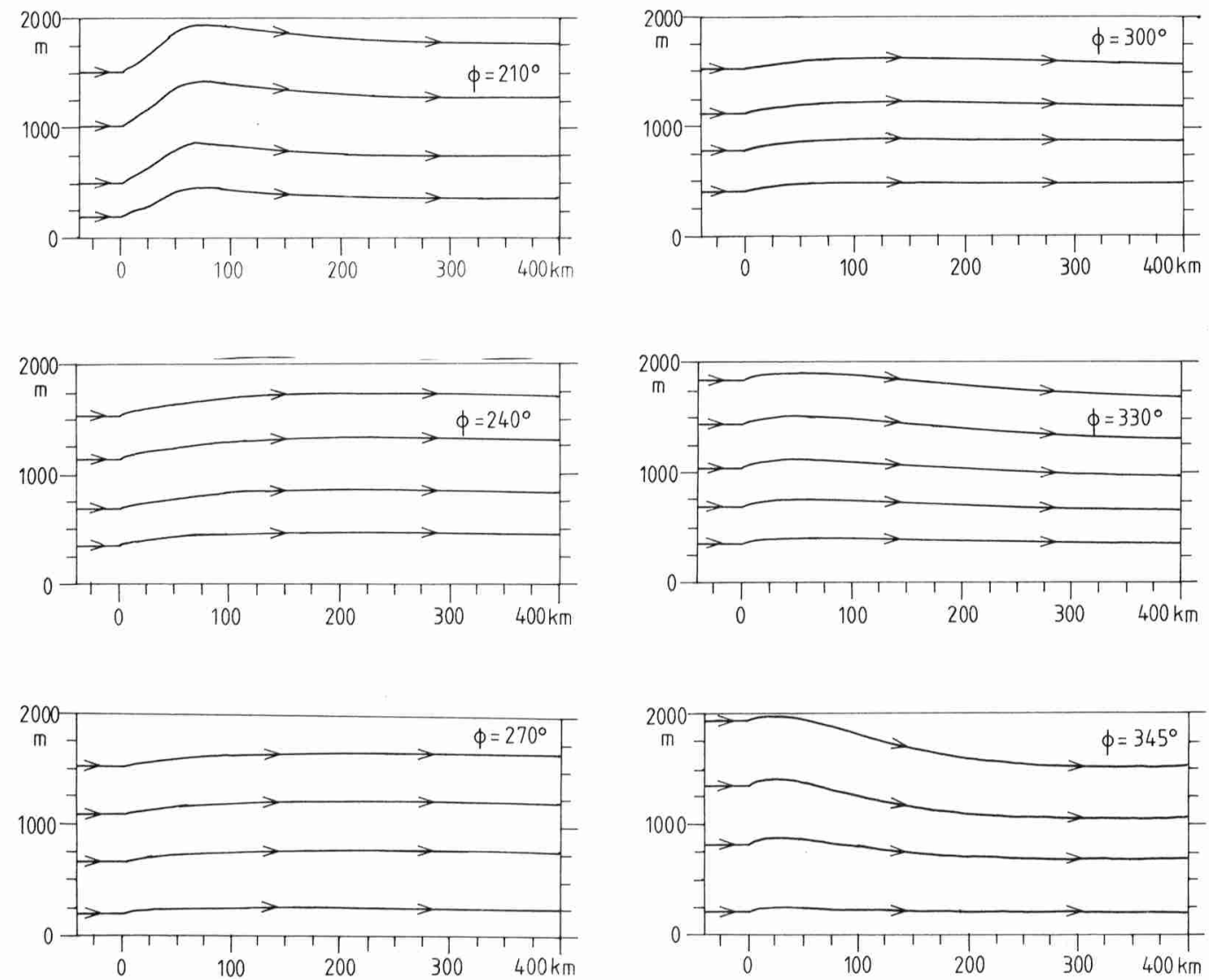

Fig. 4 (continued).

coastline. For north-northeasterly flow (type-7), marked downward motion over the coastline occurs. A distinction can be made between a pattern where the surface flow is westerly over the entire domain (type-7b), and a pattern where the surface flow changes sign. Most interesting is the secondary flow pattern occurring in southsouthwesterly geostrophic flow. The type-1 to type- 5 transition marks the point where a closed circulation over land is established. In type-6a, the easterly surface flow over land extends to $x \rightarrow \infty$, in type- 6 b over the entire domain.

We found indications that the position of maximum surface convergence (the "coastal front") does not change smoothly with the geostrophic wind direction, but jumps from type- $6 \mathrm{a}_{1}$ to type- $6 \mathrm{a}_{2}$. For a narrow range of wind directions, both types appear as a solution to the stationary problem. However, we should mention that in these cases, convergence to the final

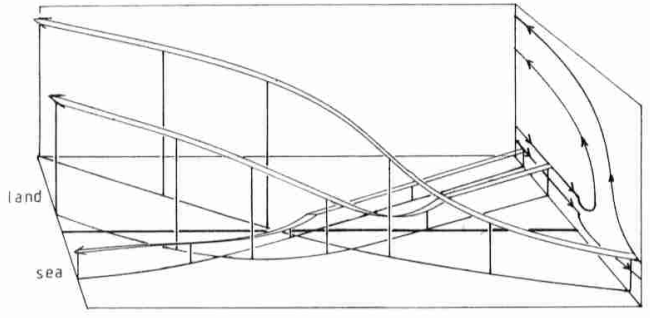

Fig. 5. A schematic 3-dimensional plot of the flow at $\phi=190^{\circ}$. The pattern in the plane at the right-hand side corresponds to Fig. 4.

solution is really very slow and sometimes irregular. It could thus be that in the time-dependent problem, the solution would linger around types $6 a_{1}$ and $6 a_{2}$, and the coastal front would shift back and forth in the vicinity of the coastline (over a typical distance of 5 to $20 \mathrm{~km}$ ). We return to this point later. 

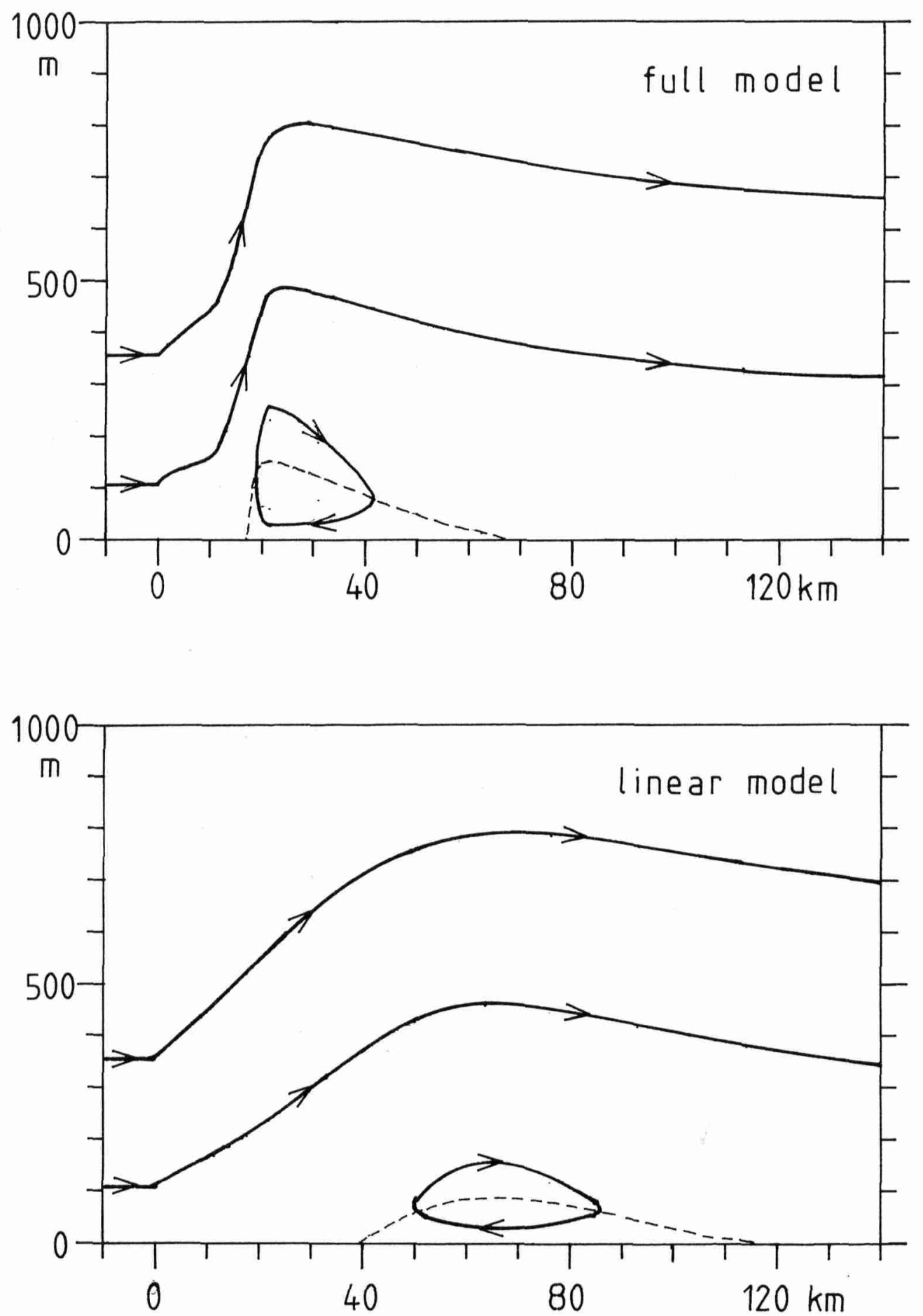

Fig. 6. Comparison between full and linear solution at $\phi=205^{\circ}$. In the area under the broken line, the flow is seaward.

Tellus 38A (1986), 5 
Since the properties of air in the lower layers normally differs over sea and land, the frictionally induced circulation in a southsouthwesterly flow helps to establish a zone of frontogenesis in the vicinity of the coastline. In Section 4, an example will be documented and discussed. At the same time, the vertical displacement of air parcels is also largest in the southsoutheasterly flow. Enhanced shower activity can therefore be expected in such situations, and observations confirm this (again, see Section 4).

A schematic three-dimensional picture of the flow for $\phi=190^{\circ}$ is shown in Fig. 5. Air parcel trajectories are projected on the bottom plane and the plane perpendicular to the coast. In this case, the along-shore component of the wind is about 10 times larger than the onshore component, both over sea and land.

In another series of experiments, the speed of the geostrophic wind was systematically varied for fixed directions. As expected, the size of the frictionally-induced circulations increases with increasing wind speed. It turned out that the increase in boundary-layer depth for stronger geostrophic wind is far more pronounced in the cases with large vertical motions. This feature finds its origin in the vertical advection of turbulent kinetic energy, i.e., the vertical redistribution of "mixing capacity". Situations with a geostrophic wind more perpendicular to the coastline show larger variations in the horizontal size of the flow patterns.

The sensitivity of the results to changes in the discontinuity in surface roughness was also investigated. A larger jump in $z_{0}$ leads to deeper and more vigorous secondary circulations. In addition to this, the transitions between the various types are found at different wind directions. Since the ageostrophic component is determined by $z_{0}$, one expects that for a decreasing step in $z_{0}$, the sectors of types-1-4 will grow at the expense of the other types. This turned out to be the case.

An interesting aspect of the solutions discussed above concerns the importance of nonlinear effects. To study this point, the equations were linearized around a reference state, being the mean of the equilibrium wind profiles over an infinite sea and continent. Symbolically,

$\boldsymbol{v}_{r}(z)=\sum_{2}^{1}\left[\boldsymbol{v}_{\text {se:a }}(z)+\boldsymbol{v}_{\text {land }}(z)\right] \quad$ for all $x$,

$$
\begin{array}{ll}
K_{r}(x, z)=K_{0}(z) & \text { for } x \leqslant 0 \\
K_{r}(x, z)=K_{1}(z) & \text { for } x>0
\end{array}
$$

Here, $K_{0}$ and $K_{1}$ are the equilibrium exchange coefficients over homogeneous sea and continent, respectively, and thus only depend on $z$.

Differences between the linearized solutions and the full solutions are generally not very large, except in south-southwesterly flow. As expected, on the basis of the previously discussed results (the suggestion of branching of the steady-state solution points to important nonlinear effects), this is understandable. An example is shown in Fig. 6, where the linear and full solution are compared for a $20 \mathrm{~m} / \mathrm{s}$ geostrophic flow from $205^{\circ}$. In the linear solution, there is an apparent horizontal smoothing of the flow pattern. However, the general dependence of the frictionally-induced flow pattern on wind direction appeared to be well explained by linear theory.

\section{An example of frontogenesis by frictional convergence}

In this section, we present an example of a socalled sw-type coastal front, i.e., a coastal front in a strong southwesterly flow caused by the interaction between frictionally-induced boundary layer convergence and convective instability. Differential heating between land and sea also contributes to the frontogenesis process, but a direct ther-

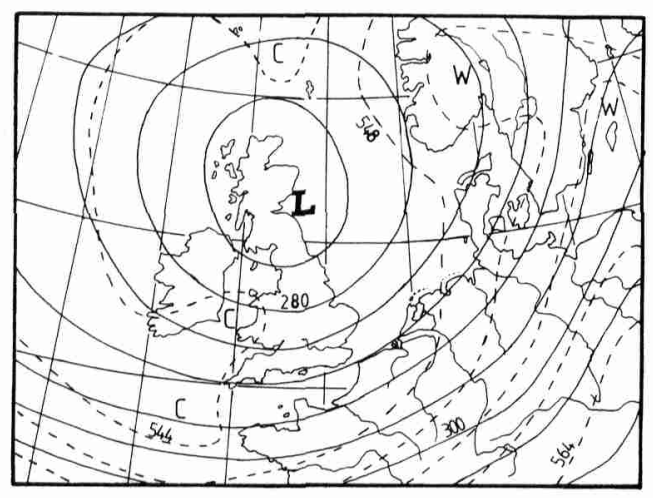

Fig. 7. Map of the $700 \mathrm{mb}$ geopotential height (solid lines) and the $1000 / 500 \mathrm{mb}$ thickness (dashed lines) at 00 GMT, 16 September 1983. Contour interval: $40 \mathrm{~m}$. 
mally driven circulation is of minor importance because of the strong winds. Strong coastal fronts appear along the Dutch coasts in polar outbreaks and are observed about 10 times per year on average. A few of these are of the type discussed here.

In Fig. 7, the $700 \mathrm{mb}$ map of 16 September 1983 is shown. A low-pressure area, centered just off the east coast of Scotland, maintains a southwesterly flow over the British Channel and the North Sea. A relatively cold air mass is advected over a warm sea and as a result thundery showers develop. On the satellite picture, Fig. 8, isolated and randomly distributed showers are present over the British Channel. On the right-hand side of the picture, clouds associated with the cold front sweeping over middle Europe are still visible. The striking feature, however, is the band of heavy showers along the coastline of Belgium and the Netherlands. Here, the flow is almost parallel to the coast. The individual showers travel along the coast towards the northeast, but the mesoscale convective system itself is quasi-stationary, as,

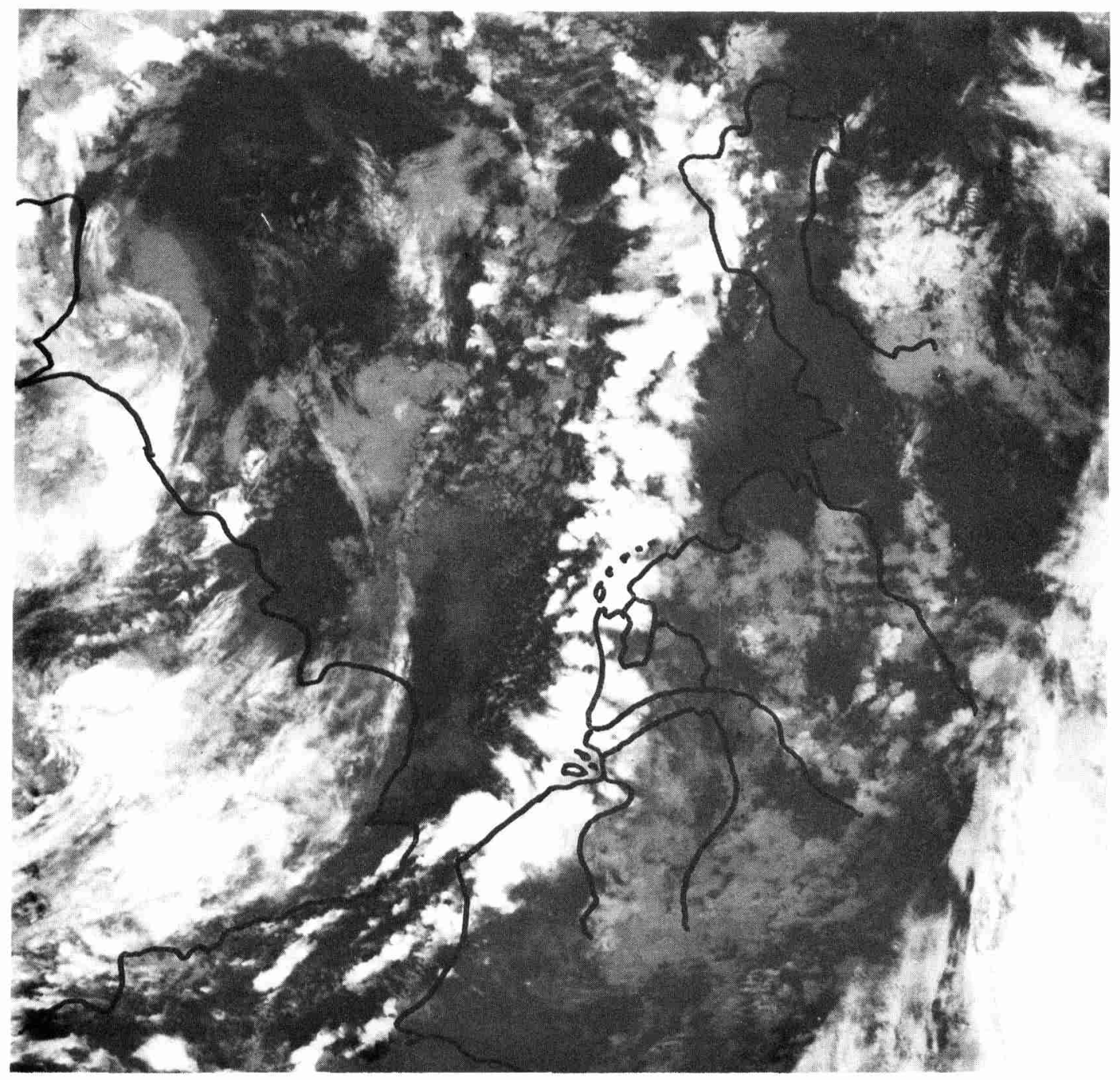

Fig. 8. NOAA-7 satellite image of the North Sea area taken on 16 September 1983,03 42 GMT

Tellus 38A (1986), 5 
for instance, can be seen on four successive radar maps (Fig. 9).

Downstream from the Dutch coast, the band of showers can be followed to the northwestern tip of Denmark. Apparently, as long as the convective system is fed with moisture and heat from the sea surface it can maintain itself. With observed winds of $15-20 \mathrm{~m} / \mathrm{s}$ and a sea-surface temperature of 16 to $17^{\circ} \mathrm{C}$, moisture fluxes were probably large. The air-sea temperature difference of $3^{\circ} \mathrm{K}$ (probably much more in the vicinity of showers) maintained the required sensible heat flux.

Over land, showers developed only during daytime. Due to this competitive convection, the organisation of the showers along the coast broke down, but was established again in the evening (not shown). Later in the night, the large-scale flow veered to a more westerly direction and the frontal character of the flow near the coast disappeared.

The type of event described above occurs a few
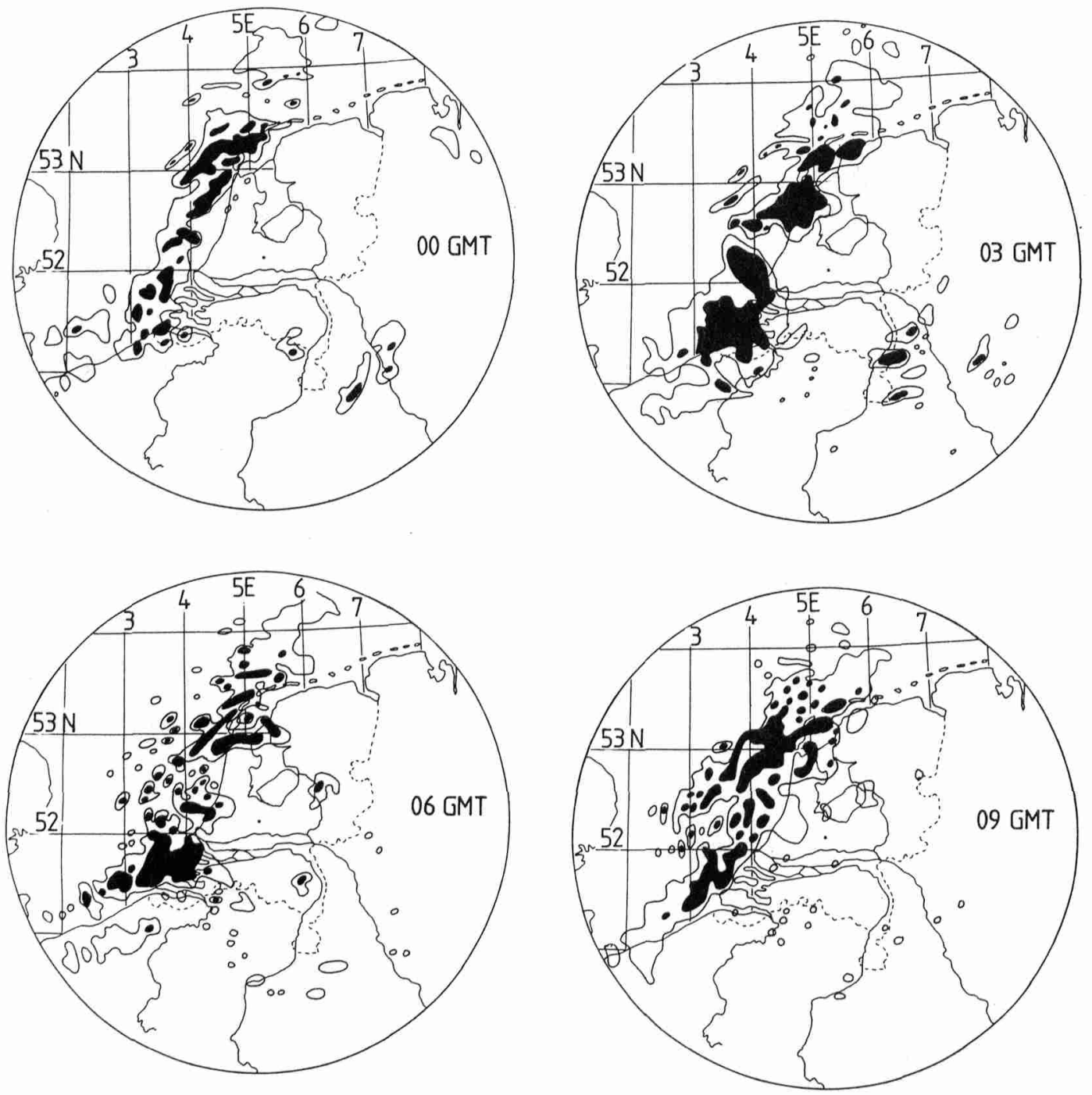

Fig. 9. Four successive radar maps based on De Bilt radar pictures at 00, 03, 06 and 09 GMT, 16 September 1983. Tops are up to $9500 \mathrm{~m}$. 
times each year. Precipitation amounts are generally large: up to $30-50 \mathrm{~mm}$ within $24 \mathrm{~h}$ or even 12 $\mathrm{h}$ in a narrow strip along the coast. The position of the precipitation maximum depends strongly on the exact direction of the gradient wind. In the heavier showers, thunder and sometimes hail occurs. In the case described above, at some locations, thunder was reported at 6 consecutive hours. It is also of interest to note that the precipitation climatology for the Netherlands shows large values in the coastal zone during fall. Although one could argue that this is just due to relatively high sea temperature, we suspect that the annual maximum of the southwesterly type of circulations in this period also plays an important rôle. Finally we would like to mention the agreement between our findings and those reported in Bergeron (1949), who showed examples of strong precipitation maxima along the Swedish east coast in a northeasterly flow.

\section{Concluding remarks}

The results discussed above have clearly shown that frictional effects at coastlines can lead to secondary circulations on the mesoscale. The southwesterly case is particularly interesting, because it favours frontogenesis and has a narrow zone with strong upward motion. Such results are generally not so obvious from currently-used mesoscale models, for two reasons. Firstly, gridpoint spacing in these models is rather large (a few $\mathrm{km}$, at least), whereas in the present calculations the spacing goes down to meters in the vicinity of the roughness discontinuity. Secondly, most existing models have smoothing operators to keep the time integration stable. In the present calculations, it appeared possible to obtain stationary flow fields without any horizontal diffusion, although it took a lot of numerical experimentation to find a stable and reliable scheme. The absence of horizontal diffusion is also the reason that projected trajectories in the various figures discussed above are not always smooth close to the coastline.

Although we did not investigate this in depth, it seems that the $l, E$ closure has a significant effect on the ultimate flow patterns. The most important process included in this way is the upward penetration of mixing capacity downstream of the roughness discontinuity. One should realize, however, that in reality stratification will play an important rôle. The stability over land and sea is generally different, which will influence the redistribution of momentum, but will also have a thermodynamic effect on the secondary circulation induced by the frictional convergence.

In a more general investigation of coastal frontogenesis (Van den Berg, 1986), it appeared that, depending on the general synoptic conditions, the land breeze can be very important in triggering coastal frontogenesis and shower activity. This result emerged from numerical experiments with a 2D-mesoscale model with detailed physics and many levels in the boundary layer. Coastal fronts of the frictional type are most active in early fall, while the land-breezeinduced type is more frequently observed in late fall and early winter. These results apply to the Dutch coast, however, and are to a large extent determined by local climatological conditions.

As a more technical conclusion, we would like finally to mention that the procedure of calculating stationary flow fields employed here is very efficient. Integrating in time until a steady state settles down takes much more computer time. The general problem of using relaxation techniques is to know whether a stable steady flow pattern occurs as a solution of the governing equations or not. In the strongly dissipative system studied in this paper, time-dependent solutions are not very likely, but one cannot be sure. Nevertheless, we feel that the procedure employed here has enabled us to make a systematic comparison of secondary flow patterns, as a function of the background geostrophic wind.

\section{Appendix A}

\section{Transformation of the equations}

Using a complex wind velocity $U=u+i v$; $i=(-1)^{1 / 2}$, and a logarithmic vertical coordinate $\zeta$, eqs. (1)-(4) become:

$$
\begin{aligned}
& \operatorname{Re} U \frac{\partial U}{\partial x}+\frac{w}{z} \frac{\partial U}{\partial \zeta}=\operatorname{if}\left(U-U_{\zeta}\right)-\frac{1}{z} \frac{\partial}{\partial \zeta} \\
& \quad \times\left(\frac{K_{m}}{z} \frac{\partial U}{\partial \zeta}\right),
\end{aligned}
$$




$$
\begin{aligned}
& \operatorname{Re} U \frac{\partial U}{\partial x}+\frac{1}{z} \frac{\partial w}{\partial \zeta}=0, \\
& \operatorname{Re} U \frac{\partial e}{\partial x}+\frac{w}{z} \frac{\partial e}{\partial \zeta}=\frac{K_{m}}{z^{2}}\left\|\frac{\partial U}{\partial \zeta}\right\|^{2}-\frac{1}{z} \frac{\partial}{\partial \zeta} \\
& \quad \times\left(\frac{K_{c}}{z} \frac{\partial e}{\partial \zeta}\right)-{\frac{(c e)^{3 / 2}}{1}}^{3} .
\end{aligned}
$$

For the advection terms, upstream, differences were used, while in all other cases, first-order derivatives are approximated by central differences. The diffusion term is approximated by:

$$
\begin{aligned}
& \frac{1}{z} \frac{\hat{c}}{\partial \zeta}\left(\frac{K}{z} \frac{\partial Q}{\partial \zeta}\right) \propto \frac{1}{z_{j} \Delta \zeta}\left[\left(\frac{K_{\mathrm{F}}}{z}\right)_{j+\frac{1}{2}} D_{\zeta} Q_{j+\frac{1}{2}}\right. \\
& \left.\quad-\left(\frac{K_{\mathrm{F}}}{z}\right)_{j-!} D_{\zeta} Q_{j-\frac{1}{2}}\right],
\end{aligned}
$$

where $D_{\zeta} Q_{j+!}=\left(Q_{j+1}-Q_{j}\right) / \Delta \zeta$. The value of $K / z$ at $\left(j+\frac{1}{2}\right)$ is the mean of the values at $j$ and $j+1$.

For computation of the vertical wind, the landward wind gradient was integrated by the trapezium rule below $550 \mathrm{~m}$, and by Simpson's rule above, because of the large grid distances. Below $1 \mathrm{~m}$, the wind profile is assumed to be linear in $\zeta$-coordinates, while $e$ has a constant value.

\section{Appendix B}

\section{Method of solution}

The equations are solved by a semi-implicit Gauss-Seidel point overrelaxation technique. Relaxation was done along columns upward, determining wind speed first and eddy diffusion thereafter. After a column, the next one is taken, and so on until the boundary of the grid, where the direction is reversed. After going from left to right and back once, the maximum absolute value of the horizontal wind correction is determined. If this is less than $10^{-4} \mathrm{~m} / \mathrm{s}$, the calculation ends.

For wind relaxation, basically a simplified version of the Newton algorithm is used, which appeared to be stable for uniformly seaward or landward flow. Where both flow directions are present, the algorithm must be stabilized. The Newton algorithm can be shown to be absolutely stable if the vertical wind is kept fixed. The continuity equation, however, causes convergence to be conditional in the sense that either horizontal grid distances should be large or the wind field should resemble the "true" solution sufficiently well. This effect is caused by the vertical acceleration, which in a number of cases affects the horizontal wind speed more quickly than advection or diffusion does. Thus, an estimation for the time scale for the growth of vertical advection is introduced which is calculated with corrections on lower grid points.

The algorithm for eddy diffusion is a modified and extended version of the Newton method. This algorithm is unstable because of singularities in the first derivative of the rest term $R$, and because of several ways of bad error propagation. For solving the problems, the difference equation is split up into terms due to horizontal advection, production, diffusion, etc. The symbolic representation reads:

$R\left(K_{i j}\right)=\sum_{k} R_{k}\left(K_{i j}\right)=0$.

Each $R_{k}$ gives a contribution to the first derivative $R^{\prime}\left(K_{i j}\right)$. The Newton algorithm reads:

$K_{\text {new }}=K_{\text {old }}-R\left(K_{i j}\right) / \sum_{k} R^{\prime}\left(K_{i j}\right)$.

The singularities in $R^{\prime}\left(K_{i j}\right)$ are caused by the contribution of the production term of $R^{\prime}$, which has a sign opposite to all other terms. Fortunately, it can be shown that the difference equation has a unique solution if negative $K$ on the grid are absent, so the singularities can be removed by changing the sign of the production term in $R^{\prime}$.

The problem of bad error propagation is solved by the method of partial growth factors. Consider the partial iteration:

$K_{i j}^{\text {new }}=K_{i j}^{\text {old }}-R\left(K_{i j}\right) / R^{\prime}\left(K_{i j}\right)$,

and give a $K_{l m}$ at a point in the vicinity of $(i, j)$ an extremely large value. Now we determine the maximal parital growth factor $g_{k}$ by

$g_{k}=\sup _{(\text {alll }, m)} \frac{K_{i j}^{\text {new }}}{K_{i j}^{\text {old }}}$.

This growth factor is used for stabilizing the Newton algorithm in the following way: 


$$
K_{\text {new }}=K_{\text {old }}-\frac{R(K)}{\sum_{k}\left[R^{\prime}(K) \cdot \max \left(1, g_{k}\right)\right]} .
$$

After this, some terms are added to the denominator in (23) in order to account for higherorder derivatives of $R$. Finally the one-dimensional wind profiles at the grid boundaries are computed by relaxation of diffusion followed by the tridiagonal algorithm for wind computation; this procedure gave results within 15 iterations. The computations are done on a CDC CYBER $180 / 855$ computer of the University of Utrecht. CP-time is about $30 \mathrm{~s}$ for uniformly landward or seaward flows, but for confluent flow types, about 5 min CP-time was required.

\section{REFERENCES}

Anthes, R. A. 1983. Regional models of the atmosphere in middle latitudes. Monthly Weather Review 111, 1306-1335.

Bergeron, T. 1949. The coastal orographic maxima of precipitation in autumn and winter. Tellus 1, 15-32.

Blackadar, A. K. 1962. The vertical distribution of wind and turbulent energy exchange in a neutral atmosphere. J. Geophys. Res. 67, 3095-3102.

Detering, H. W. 1985. Mischungsweg und Turbulenter Diffusionskoeffizient in Atmosphärischen Simulationsmodellen. Thesis, Univ. of Hannover, $211 \mathrm{pp}$.

Echols, W. T. and Wagner, N. K. 1972. Surface roughness and internal boundary layer near a coastline. J. Appl. Meteorol. 11, 658-662.

Estoque, M. A. 1962. The sea breeze as a function of the prevailing synoptic situation. J. Atmos. Sci. 19, 244-250.

$\mathrm{Ng}, \mathrm{K} . \mathrm{H}$. and Spalding, D. B. 1972. Turbulence model for boundary layer near walls. Phys. Fluids 15, 20-30.

Nieuwstadt, F. T. M. and Driedonks, A. G. M. 1979. The nocturnal boundary layer: a case study compared with model calculations. J. Appl. Meteorol 18, 13971405.

Oerlemans, J. 1980. A case study of a subsynoptic disturbance in a polar outbreak. Q.J.R. Meteorol. Soc. 106, 313-325.

Pearson, R. A., Carboni, G. and Brusasca, G. 1983. The sea breeze with mean flow. Q. J. R. Meteorol. Soc. 109, 809-830.
Pielke, R. A. 1974. A three dimensional numerical model of the sea breeze over South Florida. Mon. Wea. Rev. 102, 115-139.

Rao, K. S., Wijngaard, J. C. and Coté, O. R. 1974. The structure of the two dimensional internal boundary layer over a sudden change of surface roughness. $J$. Atmos. Sci. 31, 738-741.

Schwiesow, R. L. and Lawrence, R. S. 1982. Effects of a change of terrain height and roughness on a wind profile. Boundary Layer Meteorol. 22, 109-123.

Taylor, P. A. 1969. The planetary boundary layer above a change in surface roughness. J. Atmos. Sci. 26, 432440 .

Tennekes, H. and Lumley, J. L. 1972. A first course in turbulence. MIT Press, Massachusets.

Timmerman, H. 1963. The influence of topography and orography on the precipitation patterns in the Netherlands. KNMI Mededelingen en Verhandelingen $n r$. $80,49 \mathrm{p}$.

Van den Berg, W. D. 1986. Coastal frontogenesis in the Netherlands: observations and modelling. Ph. D. Thesis, University of Utrecht.

Walsh, J. E. 1974. Sea breeze theory abd applications. J. Atmos. Sci. 31, 2012-2019.

Yu, T. W. 1977. A comparative study on parameterization of vertical turbulent exchange processes. Mon. Wea. Rev. 105, 57-66. 